\title{
Prevalence and phylogenetic characterization of canine coronavirus from diseased pet dogs in Beijing, China
}

\author{
Shuai $\mathrm{Lu}^{1,2 \dagger}$, Di Zhang ${ }^{3 \dagger}$, Jianfang Zhou ${ }^{2}$, Zhaofei Xia ${ }^{3}$, Degui Lin ${ }^{3}$, Yongliang Lou ${ }^{1}$, \\ Wenjie $\operatorname{Tan}^{1,2^{*}} \&$ Kun Qin ${ }^{2 *}$ \\ ${ }^{l}$ School of Laboratory Medicine and Life Science, Institute of Medical Virology, Wenzhou Medical University, Wenzhou, 325035, China, \\ ${ }^{2}$ Key Laboratory of Medical Virology, National Health and Family Planning Commission, National Institute for Viral Disease Control and \\ Prevention, Chinese Center for Disease Control and Prevention, Beijing, 102206, China, \\ ${ }^{3}$ Department of Small Animal Clinical Sciences, College of Veterinary Medicine, China Agricultural University, Beijing, 100193, China
}

Received December 18,2015; accepted February 16,2016; published online May 12, 2016

Citation: Lu, S., Zhang, D., Zhou, J., Xia, Z., Lin, D., Lou, Y., Tan, W., and Qin, K. (2016). Prevalence and phylogenetic characterization of canine coronavirus from diseased pet dogs in beijing, china. Sci China Life Sci. 59, 860-862. doi: 10.1007/s11427-015-0369-x

\section{Dear Editor,}

Canine coronavirus including canine coronavirus $(\mathrm{CCoV})$ and canine respiratory coronavirus $(\mathrm{CRCoV})$ have been recognized as pathogenic viruses to dogs worldwide, causing enteric and respiratory issues (Buonavoglia et al., 2006). Zoonotic transfer of the viruses from the animal kingdom to humans has been repeatedly observed over the past decade, similarly to SARS- and MERS-coronavirus, which caused a pandemic in 2003 in China and in recent years in the Middle East, respectively (Azhar et al., 2014; Bolles et al., 2011). Thus, studies of the viral prevalence and interspecies transmissions at the animal-human interface are critically important to prepare for possible human pandemics.

In this study, a total of 246 swab samples were collected from 246 corresponding diseased pet dogs visiting the veterinary teaching hospital affiliated with the college of veterinary medicine, China Agricultural University, Beijing. Total RNA were extracted from the 246 sample swabs and subjected to screening for $\mathrm{CCoV}$ and $\mathrm{CRCoV}$ as well as canine distemper virus (CDV) and canine parainfluenza virus (CPIV) by using a reverse transcription $\mathrm{PCR}$ assay targeting the $\mathrm{N}$ gene. All

$\uparrow$ Contributed equally to this work

*Corresponding author (email: tanwj28@163.com; qkv0115@163.com)
PCR amplification products were purified and sequenced to analyze phylogenetic relationships (Table S1 and S2). Data were analyzed by the chi-squared test using SAS software version 9.2 (SAS Institute, Inc., USA). $P<0.05$ was considered statistically significant.

Infections with $\mathrm{CCoV}$ were detected in 64 of the 246 surveyed samples, exhibiting the highest infection rate of $26.0 \%$ (95\%CI: $19.3 \%-31.9 \%)$, followed by 40 for CDV $(16.3 \%$, 95\%CI: $11.2 \%-21.3 \%), 16$ for $\mathrm{CRCoV}(6.5 \%, 95 \% \mathrm{CI}$ : $3.3 \%-9.7 \%)$, and 9 for CPIV $(3.7 \% 95 \% \mathrm{CI}: 1.3 \%-6.0 \%)$. Pets aged from several days to many years were infected with the indicated viruses, while $75.2 \%$ of the infections mainly occurred in puppies less than 12 months old. According to medical records, the ages of $\mathrm{CCoV}$ - and $\mathrm{CRCoV}$-infected dogs were generally less than three months $(P<0.05)$, while there was no difference in CDV and CPIV infections. No significant differences were observed regarding gender (Table S3). Notably, it was clear that cases infected with $\mathrm{CCoV}$ predominately manifested as gastrointestinal signs, while CDV infections exhibited respiratory symptoms $(P<0.05)$. $\mathrm{CCoV}$ was predominately examined from rectal swabs, while CDV- and CPIV-positive specimens were mainly found in respiratory tracts $(P<0.05)$ (Table S3). Co-infections were also discovered in a single case, $\mathrm{CCoV}$ and $\mathrm{CDV}$ co-infections were found in nine cases, and four dogs showed $\mathrm{CCoV}$ 
and $\mathrm{CRCoV}$ co-infection, while other co-infection patterns were rarely observed.

Nucleotide sequences in this study were deposited in the Genbank (http://www.ncbi.nlm.nih.gov/genbank) under the accession NO. KR265176-KR265207. Phylogenetic analysis was performed using a portion of $\mathrm{N}$ gene partial sequences derived from the positive samples. As coronavirus has been shown to be capable of crossing the species barrier to infect human and cause pandemics, we determined the nearly full-length sequences of the $\mathrm{N}$ gene of canine coronavirus. Notably, the $\mathrm{N}$ gene sequences of the coronavirus were highly divergent. Phylogenetically, these sequences were divided into three distinct groups. Group 1 included two clear clusters: strains CCoV/BJ-84, 117, 147, 151,161, 171, 174, 176, and 184 grouped closely with the strain detected from the Chinese ferret-badger (Melogale moschata) in the wet market and other CCoVs from North America, while CCoV/BJ-87 and 145 fell into another cluster and grouped together with European isolates such as CCoV/23-03/Italy/2004. The N gene of CRCoV/BJ-34, 202, and 221 in group 2 was very similar to that of a South Korea isolate, which also clustered with HCoV-OC43 (Figure 1).

Even the gentlest of our friendly pets can harbor harmful pathogens to both animals and humans. It is necessary to closely evaluate the zoonotic pathogens transferred to other animal hosts or human populations; thus, the best way to prevent and control potential zoonotic infectious diseases is to understand the ecology and evolution of these viruses. Our study examined the prevalence of $\mathrm{CCoV}$ and $\mathrm{CRCoV}$ as well as the phylogenetic characterization. $\mathrm{CCoV}$ showed the highest detection rate among the four viral pathogens, which different from previously reported rates (Erles et al., 2004; Wang et al., 2016). This may be because of the viral spatiotemporal distribution and varied vaccination protocols among regions. Given the complex ecology of viral diseases in pet dogs, many pathogens such as canine parvovirus (CPV), canine adenovirus (CAV), and other common infectious agents were not examined. Thus, co-infections were rarely observed among these investigated viruses. As a nation-wide compulsory vaccination program against coronavirus has not been implemented, the different immunization status of companion animals may select genetic or antigenic variants, leading to the emergence of novel viral strains. Because the $\mathrm{N}$ gene was highly conserved among the coronavirus family, segregation of the $\mathrm{N}$ gene in the phylogenetic tree implied the genetic diversity of $\mathrm{CCoV}$ in this region. Such an ecological niche may provide an optimal opportunity to generate novel viruses with the potential for interspecies transmission, similarly to the emergence of SARS-CoV and MERS-CoV originating in the animal population. However, with increases in the Chinese pet dog population and increasing contact with human population in recent years, new ecology of viral pathogens in canines will

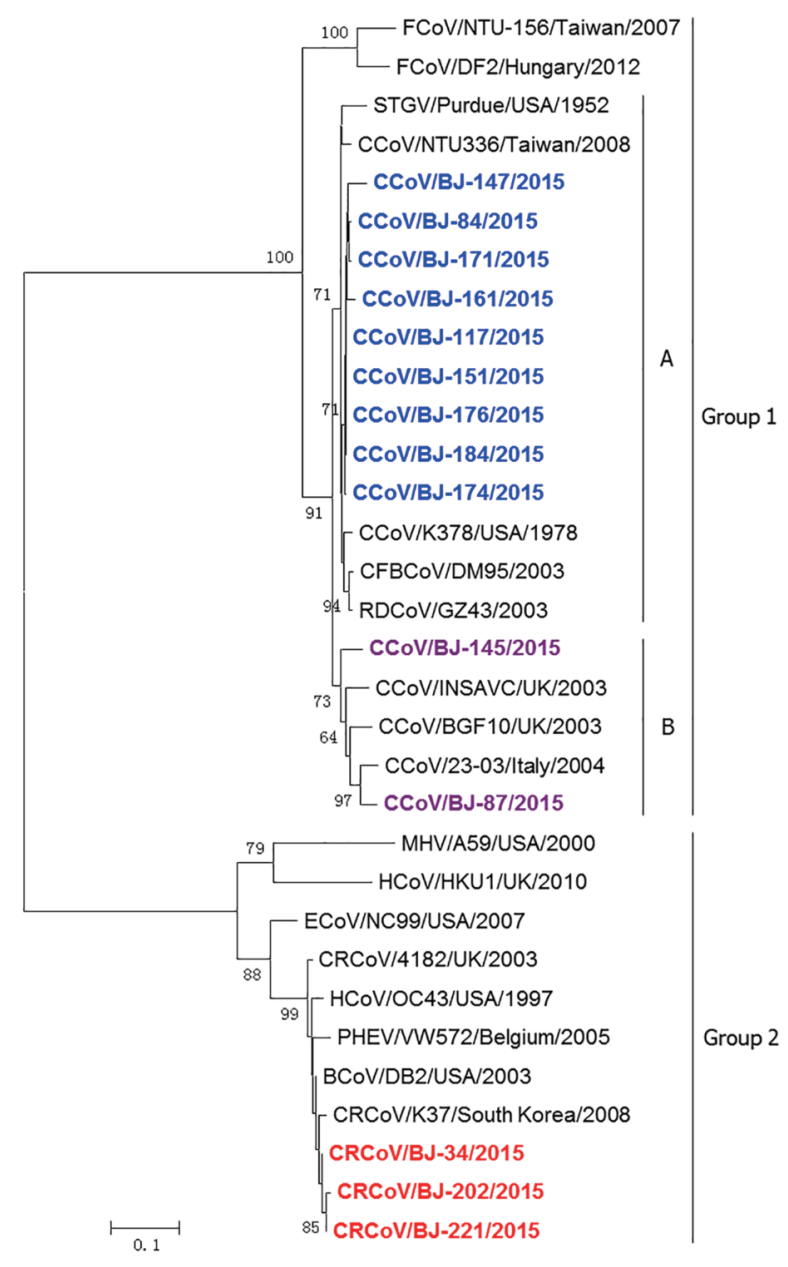

Figure 1 Phylogenetic relationships between $\mathrm{N}$ gene sequence of $\mathrm{CCoV}$ and $\mathrm{CRCoV}$. The tree was generated by the neighbor-joining method using the MEGA6 program. Values above the branches are neighbor-joining bootstrap values from 1,000 replicates. Analysis was based on the nearly full-length $\mathrm{N}$ gene. Scale bar, 0.1 nucleotide substitution per site. Abbreviations: CFBCoV, Chinese ferret badger coronavirus; RDCoV, raccoon dog coronavirus; STGV, swine transmissible gastroenteritis virus; MHV, murine hepatitis virus; ECoV, equine coronavirus; PHEV, porcine hemagglutinating encephalomyelitis virus; $\mathrm{BCoV}$, bovine coronavirus.

be more complex and elusive. Therefore, it is necessary to strengthen comprehensive surveillance in companion animals in order to prepare for emerging and re-emerging diseases.

Compliance and ethics The author(s) declare that they have no conflict of interest.

Acknowledgements This work was supported by the State Megaproject for Infectious Disease Research of China (2014ZX10004001-002, 2013ZX10004101, 2013ZX10004601, 2014ZX10004002-001-004) and the Teachers' Seed Fund for Research (2014XJ008).

Azhar, E.I., El-Kafrawy, S.A., Farraj, S.A., Hassan, A.M., Al-Saeed, M.S., Hashem, A.M., and Madani, T.A. (2014). Evidence for camel-to-human transmission of MERS coronavirus. N Engl J Med 370, 2499-2505.

Bolles, M., Donaldson, E., and Baric, R. (2011). SARS-CoV and emergent coronaviruses: viral determinants of interspecies transmission. Curr 
Opin Virol 1, 624-634

Buonavoglia, C., Decaro, N., Martella, V., Elia, G., Campolo, M., Desario, C., Castagnaro, M., and Tempesta, M. (2006). Canine coronavirus highly pathogenic for dogs. Emerg Infect Dis 12, 492-494.

Erles, K., Dubovi, E.J., Brooks, H.W., and Brownlie, J. (2004). Longitudinal study of viruses associated with canine infectious respiratory disease. J
Clin Microbiol 42, 4524-4529.

Wang, X.Y., Li, C.Q., Guo, D.H., Wang, X.Y., Wei, S., Geng, Y.F., Wang, E.Y., Wang, Z.H., Zhao, X.W., Su, M.J., Liu, Q.J., Zhang, S.Y., Feng, L., and Sun, D.B. (2016). Co-circulation of canine coronavirus I and $\mathrm{IIa} / \mathrm{b}$ with high prevalence and genetic diversity in Heilongjiang province, Northeast China. PLoS One 11, e0146975.

Open Access This article is distributed under the terms of the Creative Commons Attribution License, which permits any use, distribution, and reproduction in any medium, provided the original author(s) and source are credited.

\section{SUPPORTING INFORMATION}

Table S1 Primers used in this study

Table S2 Strains of coronavirus used for phylogenetic analysis

Table S3 Demographic and epidemiological data in this study

The supporting information is available online at life.scichina.com and link.springer.com. The supporting materials are published as submitted, without typesetting or editing. The responsibility for scientific accuracy and content remains entirely with the authors. 